\title{
Risks to the Infant Relating to Techniques of Local Anesthesia
}

\section{H. Pedersen, M. Finster}

Those risks related to regional anesthesia in obstetrics which affect the infant may derive from two sources: those arising indirectly, through maternal complications and/or diminished uteroplacental blood flow, or directly, through the effects of local anesthetic drugs, or their metabolites, on the fetus and newborn.

1) Indirect Effects.

It is obvious that maternal convulsions resulting from an overdose or inadvertent intravascular injection of a local anesthetic may lead to fetal asphyxia. Paralysis of the mother's respiratory muscles by high epidural or spinal anesthesia may also result in maternal, and consequently fetal hypoxia. However, these complications are fortunately infrequent in competent hands.

A more common problem associated with epidural and spinal anesthesia is maternal hypotension. This is due to sympathetic blockade, affecting the arterial as well as the venous circulation. Arterial relaxation will lower the peripheral resistance, while venous relaxation tends to produce peripheral pooling of blood, and reduction in cardiac output. The resulting hypotension may diminish the placental perfusion sufficiently to cause fetal asphyxia. This phenomenon may be aggraved by vena caval and aortic compression, if the parturient is placed in the supine position (2). The incidence and severity of maternal hypotension can be greatly reduced by rapidly infusing at least 1 liter of a crystalloid solution (Ringer's lactate) prior to induction of anesthesia. Hypotension, if it occurs, despite both this precaution and proper positioning of the mother (left uterine displacement), should be promptly corrected by administration of a small intravenous dose of a predominantly $\beta$-adrenergic vasopressor (e.g., ephedrine, $10-15 \mathrm{mg}$ ). $\alpha$-adrenergic drugs, even though capable of correcting maternal hypotension, may further aggravate fetal asphyxia by constricting the uteroplacental vasculature (3).

Epinephrine-containing local anesthetics, when used in obstetrics, may also affect the fetus adversely by the mechanism just described, namely, uteroplacental vasoconstriction. When epinephrine, in small doses, is infused into the pregnant ewe, there is a dose-dependent decrease in uterine blood flow (13). Consequently, there is a consensus among obstetric anesthesiologists in the U.S.A. that only plain solutions of local anesthetics should be used in the parturient.

Unfortunately, local anesthetics injected into the epidural or paracervical space, even without added epinephrine or maternal hypotension, may cause transient placental hypoperfusion and fetal bradycardia. This phenomenon is currently attributed to an increase in uterine activity and/or vasoconstriction of the uterine arteries, which has been noted when high concentrations of local anesthetics were achieved in the uterine vessels of experimental animals; e.g., $5-23 \mu \mathrm{g} / \mathrm{ml}$ of lidocaine (5), or in "in vitro" experiments (6). These high concentrations are probably reached in the vicinity of the uterine arteries following induction of

0300-5577/81/0091-0015\$2.00 
paracervical block.

However, low blood concentrations of local anesthetics which are commonly encountered following epidural anesthesia may also lead to a transient reduction in uterine blood flow. This conclusion is based on a recent study carried out in our laboratory, using chronically instrumented pregnant sheep, which has shown that lidocaine infused intravenously to the mother for sixty minutes produced an immediate rise in uterine activity and reduction in uterine blood flow to $30-35 \%$ below control, both of which effects began to return to control values after five minutes (9). The infusion rates were such as to produce maternal drug levels of $2-4 \mu \mathrm{g} / \mathrm{ml}$ and fetal levels of $1-2 \mu \mathrm{g} / \mathrm{ml}$. These findings may explain the occasional transient fetà 1 bradycardia seen shortly after epidural injection of local anesthetic in the parturient in the absence of hypotension or intravascular injection.

\section{2) Direct Effects}

As has been known since the mid-1960's, local anesthetics and their metabolites cross the placenta readily, and may exert direct untoward effects in the fetus and newborn. More recently it has been shown that infants born to mothers who have received either lidocaine or mepivacaine for analgesia in labor and delivery, even though vigorous at birth, achieve poorer scores than infants of unmedicated mothers in neurobehavioral studies carried out in the first day of life (14).

Administration of amide-type local anesthetics by repeated injection, as in the continuous epidural technique, may lead to accumulation of relatively high concentrations of drug in maternal and fetal blood and neonatal depression $(10,8)$. Therefore, the risk of overdose should be minimized by using the lowest amount and concentration necessary to obtain the desired analgesia for any given drug. Accumulation may also be limited by the use of long-acting drugs, like bupivacaine, which require less frequent re-injection, or by the use of rapidly-metabolized drugs, such as 2-chloroprocaine. Chloroprocaine is an ester, hydrolyzed by plasma pseudocholinesterase. Our in-vitro measurements of the half-lives of this

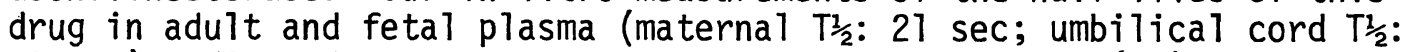
$43 \mathrm{sec}$ ) indicated an extremely rapid rate of hydrolysis (11). This explains why only nanogram concentrations have been found in maternal blood for 5 - 10 minutes after epidural injection, and only a metabolite can be detected thereafter (7). Chloroprocaine and bupivacaine result in most favorable neurobehavioral responses in the newborn and are the most commonly used local anesthetics in the U.S.A.

Prilocaine (Citanest) is another amide-type drug, similar to lidocaine in potency, onset and duration of action, but half as toxic. However, its metabolite, orthotoluidine, oxidizes hemoglobin to methemoglobin. While methemoglobinemia has been known to occur in both mother and fetus, the intravenous administration of methylene blue $2 \mathrm{mg} / \mathrm{kg}$ will lead to prompt reversal in both mother and fetus (12).

Fetal intoxication is more likely to occur if the fetus is acidotic prior to maternal medication. This has been illustrated in a recent report on 4 infants born severely acidotic, the $\mathrm{pH}$ of the umbilical artery varying from 7.02 to 7.16 (1). Three of the mothers had received mepivacaine, and one lidocaine, for epidural anesthesia. The drug concentration ratios between umbilical vein and maternal vein at birth (UV/MV), which 
ranged from 1 to 3 , were substantially higher than those found in normal newborns, where UV/MV ratios for mepivacaine and lidocaine lay between 0.5 and 0.75 . These relatively high drug concentrations in the fetal blood can be explained by "ionic trapping" in the more acid medium. An identical phenomenon undoubtedly occurs in acidotic fetal tissues. Therefore, extra caution should be exercised when administering local anesthetics to a parturient known to have an asphyxiated fetus.

Finally, another fetal risk is direct injection of a local anesthetic into the presenting part. This can complicate attempted caudal, paracervical or even pudendal block. Prevention requires alertness and strict adherence to recommended techniques. The intoxicated newborn is flaccid, apneic and exhibits persistent bradycardia even when well-oxygenated (4). Tonic-clonic convulsions occur within minutes, or a few hours, after delivery. Treatment consists of supportive measures, as well as diuresis and gastric lavage to promote drug elimination.

\section{References}

1. Brown, W.U., Jr., Be11, G.C., Alper, M.H.: Acidosis, local anesthetics and the newborn. Obstet. Gynecol. 48 (1976) 27.

2. Eckstein, K.L., Marx, G.F.: Aortocaval compression and uterine displacement. Anesthesiology, 40 (1974) 92.

3. Eng, M., Berges, P.U., Ueland, K., et a1: The effects of methoxamine and ephedrine in normotensive pregnant primates. Anesthesiology, 35 (1971) 354 .

4. Finster, M., Poppers, P.J., Sinclair, J.C., et al: Accidental intoxication of the fetus with local anesthetic drug during caudal anesthesia. Am. J. Obstet. Gynecol. 92 (1965) 922.

5. Fishburne, J.L., Jr., Greiss, F.C., Jr., Hopkinson, R., et al: Responses of the gravid uterine vasculature to arterial levels of local anesthetic agents. Am. J. Obstet. Gynecol. 133 (1979) 753.

6. Gibbs, C.P., Noel, S.C.: Response of arterial segments from gravid human uterus to multiple concentrations of lignocaine. $\mathrm{Br}$. J. Anaesth. 49 (1977) 409.

7. Kuhnert, B.R. Personal communication.

8. Moore, D.C., Bridenbaugh, L.D., Bagdi, P.A., et al: Accumulation of mepivacaine hydrochloride during caudal block. Anesthesiology, 29 (1968) 585.

9. Morishima, H.0., Gutsche, B.G., Stark, R.I., et a1: Relationship of fetal bradycardia to maternal administration of lidocaine in sheep. Am. J. Obstet. Gynecol. In press.

10. Morishima, H.0., Danie1, S.S., Finster, M., et al: Transmission of mepivacaine hydrochloride (Carbocaine) across the human placenta. Anesthesiology, 27 (1966) 147.

11. O'Brien, J.E., Abbey, V., Hinsvark, 0., et a1: Metabolism and measurement of chloroprocaine, an ester-type local anesthetic. $J$. Pharm. Sci. 68 (1979) 75.

12. Poppers, P.J., Finster, M.: The use of prilocaine hydrochloride (Citanest) for epidural analgesia in obstetrics. Anesthesiology, 29 (1968) 1134.

13. Rosenfeld, C.R., Barton, M.D., Meschia, G.: Effects of epinephrine on distribution of blood flow in the pregnant ewe. Am. J. Obstet. Gyneco1. 124 (1976) 156.

14. Scanlon; J.W., Brown, W.U., Jr., Weiss, J.B., et al: Neurobehavioral responses of newborn infants after maternal epidural anesthesia. Anesthesiology 40 (1974) 121. 